\title{
XI International Conference "Stability and Oscillations in Nonlinear Control Systems" (Pyatnitskii Conference)
}

DOI: $10.1134 /$ S0005117911090013

This special issue contains selected papers prepared from the proceedings of XI International Conference "Stability and oscillations in nonlinear control systems" (Pyatnitskii conference) that took place on June 1-4, 2010, at the Trapeznikov Institute of Control Sciences of the RAS with participation of the Department of Energetics, Machine Engineering, Mechanics, and Control Processes of the RAS and the Scientific Council on the theory of Controllable Processes and Automation of the RAS.

The objective of this conference is to provide researchers and specialists with an opportunity to meet and discuss with colleagues their new results and original approaches and establish contacts for further collaboration on all aspects of stability, control, and dynamics theory.

The conference had 349 participants from Russia and other countries (Armenia, Belarus, Kazakhstan, Ukraine, Uzbekistan, Australia, Bulgaria, Germany, Egypt, Israel, Spain, Mexico, Slovakia, USA, France) who delivered 349 talks. The conference united most of the Russian-speaking researchers in the field.

The basic research directions of the conference are as follows: fundamental problems of stability theory and motion stabilization; fundamental problems and methods of nonlinear oscillations theory; Lyapunov functions method for nonlinear control systems and the Hamilton-Jacobi-LyapunovBellman approach in optimal control theory and in game control problems; smooth and nonsmooth dynamics; controllability and observability problems for control systems; robust control problems; control for mechanical systems; stability and control for hybrid systems and systems with switchings; applied control problems and computer methods. Under the general direction of "Smooth and Nonsmooth Dynamics," a mini-symposium "Dynamics of Nonsmooth Systems" was organized; under "Fundamental Problems and Methods of Nonlinear Oscillations Theory," a special meeting "Analytic-numerical Methods for Studying Oscillations" especially for young researchers.

The scientific program consisted of 3 plenary sessions, 18 sectional sessions, 3 sessions on the mini-symposium, and also a panel discussion. The program contained 10 45-minute invited talks, 14 30-minute lectures on the scientific directions of the conference, 195 20-minute talks, and 25 30-minute talks on the mini-symposium.

Papers prepared from the talks of the conference are also published in other issues of "Automation and Remote Control" and also in the journals "Applied Mathematics and Mechanics," "Control Theory and Systems," etc. Extended abstracts of all talks included in the conference program have been published in an electronic form in two working languages of the conference on http://www.stab10.ru/; this Web site also contains all information relevant for the conference.

The conference was supported by the Russian Foundation for Basic Research, project no. 1001-06036-g and the Department of Power Engineering, Machine Building, Mechanics, and Control Processes, Russian Academy of Sciences.

Tkhai V.N. 